\title{
How Do We Manage Hematopoietic Cell Transplant during the SARS-CoV-2 Pandemic?
}

\author{
Haowen Xiao ${ }^{a, b}$ Yi Luo ${ }^{b, c}$ Jimin Shi ${ }^{b, c}$ Aiyun Jin ${ }^{b, c}$ Mohamad Mohty ${ }^{d}$ \\ Arnon Nagler $^{e}$ Robert Peter Gale ${ }^{f}$ He Huang ${ }^{b, c}$ \\ aDepartment of Hematology, Sir Run Run Shaw Hospital, Zhejiang University School of Medicine, Zhejiang Province, \\ PR China; 'bnstitute of Hematology, Zhejiang University, Hangzhou, Zhejiang Province, PR China; 'Bone Marrow \\ Transplantation Center, The First Affiliated Hospital, Zhejiang University School of Medicine, Hangzhou, Zhejiang \\ Province, PR China; 'Sorbonne Université, Saint-Antoine Hospital, AP-HP, and INSERM UMRs 938, Paris, France; \\ 'Chaim Sheba Medical Center, Tel-Hashomer, Sackler School of Medicine, Tel Aviv University, Ramat Gan, Israel; \\ ${ }^{f}$ Research Department of Immunology and Inflammation, Centre for Haematology, Imperial College London, \\ London, UK
}

\section{Keywords}

Hematopoietic cell transplant · Severe acute respiratory syndrome coronavirus- 2 - Coronavirus infectious disease 2019. Donor

\begin{abstract}
Patients receiving a hematopoietic cell transplant are thought to be at increased risk of infection with severe acute respiratory syndrome coronavirus-2 (SARS-CoV-2) and coronavirus infectious disease 2019. Transplant activities at our center continue, and notably, no patient has been infected with SARS-CoV-2. Indeed, social distancing, masking, and education for patients and donors are major pillars of prevention. We recommend potential transplant recipients and donors to be tested for SARS-CoV-2 with qRT-PCR, serum antibody detection, and a lung CT scan pretransplant. If possible, stem cells from HLA-matched unrelated donors by local processing laboratories should be cryopreserved and shipped before initiating pretransplant conditioning. An alternative HLA-haplotype-matched related donor should be identified and evaluated as a backup. The interval immediately after discharge is the time of greatest risk for SARS-
\end{abstract}

CoV-2 infection because of travel and exposure to infected persons. We recommend self-isolation and minimal contact with family members. Nonessential clinic visits should be deferred or substituted with telemedicine consultations if possible. These recommendations are based on our experience at a major transplant center in China. Although some recommendations are evidence based, other recommendations are not and warrant validation in controlled trials.

(c) 2021 S. Karger AG, Basel

\section{Introduction}

Cancer patients are at an increased risk of severe infections because of the cancer and bone marrow and immunosuppressive therapies. Patients with hematologic malignancies are particularly susceptible to infection, and recipients of a hematopoietic cell transplant (HCT) are at an even higher risk [1]. Whether this increased risk also applies to severe acute respiratory syndrome coronavirus-2 (SARS-CoV-2) infection is controversial but is likely [2-6]. One study reported a similar case rate of SARSCov-2 infections but a higher case fatality rate in hospital- karger@karger.com www.karger.com/aha

(C) 2021 S. Karger AG, Base

Karger'
He Huang

Bone Marrow Transplantation Center, The First Affiliated Hospital Zhejiang University School of Medicine, No. 79 Qingchun Rd. Zhejiang Province, Hangzhou 310003 (China)

huanghe@zju.edu.cn 
ized patients with hematologic malignancies [7]. Another study reported a higher case rate but a low case fatality rate in patients with CML [8]. Recently, data from the Memorial Sloan Kettering Cancer Center indicated that $40 \%$ of 423 patients with neoplasms were hospitalized for coronavirus infectious disease 2019 (COVID-19) illnesses, $20 \%$ developed severe respiratory illnesses, including 9\% who required mechanical ventilation, and 9\% died [9]. Overall, COVID-19 illnesses are associated with higher rates of hospitalization and severe outcomes in patients with cancer. The SARS-CoV-2 pandemic will likely impact HCT activity globally. Based on experience from our transplant center, we suggest strategies to reduce the risk of SARS-CoV-2 infection. To date, there is a lack of evidence on COVID-19 in the HCT setting to make formal guidelines.

\section{Prevalence of COVID-19 in Patients with Hematologic Disorders}

There are 29 patients with hematologic disorders and COVID-19 infections in Wuhan city, which is a community with a high prevalence of COVID-19, as follows: one child with ALL [10]; 28 adults including one adult with aplastic anemia [11]; 4 adults with CML [8]; 2 adults with myelodysplastic syndrome; 5 adults with plasma cell myelomas; and 16 adults with ALL or AML including 2 who were posttransplant $[7,12]$. In communities with a low prevalence, only 1 patient with CLL with COVID-19 has been reported [13]. The case fatality rate in patients with hematologic diseases and SARS-CoV-2 infections is high, with 6 deaths among 11 patients [11]. A similar high case fatality rate has been reported by others with 8 deaths among 13 patients with hematologic cancers and $\mathrm{CO}$ VID-19 infections [7].

Studies with a focus on the prevalence and clinical features of SARS-CoV-2 infection in patients receiving HCTs or other cell therapies are limited. Indeed, there is heightened concern for these patients for the following reasons: (1) pre- and post-transplant immune suppression; (2) movement of healthy donors through geographic areas with different community-based infection rates and through cities, countries, and regions which may have imposed travel restrictions; (3) the likelihood of increased risk after discharge because of frequent visits from family members, frequent hospital visits, and blood testing; (4) shortage of blood and blood products; and (5) uncertainty over travel restrictions and quarantines which affect transplant planning.

Management of Transplant during SARS-CoV-2

\section{Transplant Activity at Our Center during the SARS- CoV-2 Outbreak}

The First Affiliated Hospital of Zhejiang University School of Medicine (FAHZU) in Hangzhou, Zhejiang province, is one of the 2 hospitals designated to treat persons with severe COVID-19 in Zhejiang province, China. The first patient was admitted on 19 January 2020. As of 18 March 2020, a total of 105 patients with confirmed COVID-19 infections were admitted to our hospital, including 79 with severe and critically severe disease (see grading below). Confirmed cases were based on exposure history (including cluster transmission), clinical manifestations (fever and respiratory symptoms), lung computed tomography (CT) scan findings, results of SARS-CoV-2 nucleic acid detection by qRT-PCR, and immune assay detection of anti-SARS-CoV-2 antibodies. COVID-19 severity was graded as follows [14]: (1) mild (mild clinical symptoms and no sign of pneumonia on chest CT); (2) moderate (fever and respiratory symptoms and signs of pneumonia on chest CT); (3) severe (respiratory rate $\geq 30$ / min, oxygen saturation $\leq 93 \%$ at rest, and/or ratio of arterial oxygen partial pressure to fractional inspired oxygen $\leq 300 \mathrm{~mm} \mathrm{Hg}\left[\mathrm{Pa}_{\mathrm{O}_{2}} / \mathrm{F}_{\mathrm{IO} 2}\right]$ ); and (4) critically severe (respiratory failure requiring mechanical ventilation, shock, and/or other organ failure needing intensive care unit monitoring and therapy. No staff member is known to have been infected. FAHZU has published a handbook on how to prevent and treat SARS-CoV-2 infection and COVID-19 [15].

Between 24 January 2020, and 15 March 2020, we only performed transplants in patients who were already admitted to a laminar airflow clean ward. Over the past 3 months, 49 patients received a transplant, including 39 from an HLA-haplotype-matched related donor, 4 from HLA-identical siblings, 1 from an HLA-matched unrelated donor, and 5 autotransplants. No patient has become infected with SARS-CoV-2.

\section{How to Test Recipients Pretransplant}

If your area has a high community prevalence of SARSCoV-2 infection, as defined by local health authorities, the number of infected persons may exceed the local hospital bed capacity and the number of healthcare providers. If possible, transplants were deferred after weighing the risks of delay and disease progression, as well as consideration of alternative therapies. If a transplant could not be postponed, we recommended screening the recip-

Acta Haematol 2021;144:500-506 501 
ient and donor for SARS-CoV-2 infection, reviewed the transplant procedure, and secured access to the graft if coming from elsewhere. For areas with a low community prevalence of SARS-CoV-2, which should be determined by taking in account reports from local health authorities, reviewing the latest data, and whether home sheltering, social distancing, home quarantining, and transplant units operate normally.

\section{Pretransplant Recommendations}

\section{Testing Recipients for SARS-CoV-2 Infection by \\ qRT-PCR and Lung CT Scan}

The 7th version of the Clinical Guidance for Diagnosis and Treatment for COVID-19 Pneumonia, issued by the National Health Commission of China, states that SARSCoV-2 may be transmitted via respiratory aspirates, droplets, feces, and aerosols [14, 16]. The European Society for Blood and Marrow Transplantation (EBMT) recommends that potential transplant recipients should home isolate for 14 days pretransplant [17].

Between 20 and $50 \%$ of individuals with SARS-CoV-2 infection are asymptomatic, but this is likely a substantial underestimate because the number of individuals infected with SARS-CoV-2 is usually unknown unless there is population-based testing of asymptomatic individuals $[15,18,19]$. These asymptomatic individuals are the major source of spread of SARS-CoV-2, with an estimated $R_{0}$ of 2.5-3 [20]; between 15 and $20 \%$ develop severe COVID-19 [16, 21]. Thus, we recommend that potential transplant recipients be tested for SARS-CoV-2 by qRTPCR and should have $\geq 2$ consecutive negative tests $\geq 24 \mathrm{~h}$ apart 7-14 days before the transplant to prevent/avoid unexpected exposures (i.e., limit contacts, minimize community exposures, and wearing a mask) in the interval.

A lung CT scan should be considered for all recipients pretransplant. The China Medical Treatment Expert Group for COVID-19 analyzed 1,099 hospitalized patients with laboratory-confirmed SARS-CoV-2 infections from 552 hospitals through 29 January 2020. Groundglass opacity and bilateral patchy shadowing were the most common findings on lung CT scan in patients with COVID-19; however, no radiologic abnormalities were demonstrated in approximately $20 \%$ of patients with nonsevere disease and in $<5 \%$ of patients with severe disease [21]. With qRT-PCR results as a reference in 1,014 patients, $60-93 \%$ of the patients initially had positive CT findings consistent with COVID-19 before or simultaneous to the initial positive qRT-PCR results; the lung CT scan was reported to have a diagnostic sensitivity of $97 \%$, a positive predictive value of $65 \%$, and a negative predictive value of $83 \%$ [22]. The following laboratory procedures should be performed on all potential transplant recipients.

Although qRT-PCR for SARS-CoV-2 for naso- or oropharyngeal swabs, sputum, and lower respiratory tract samples is widely used and recommended in the 6th version of the Clinical Guidance for Diagnosis and Treatment for COVID-19 Pneumonia from the National Health Commission of China, qRT-PCR has limitations, such as a short detection window from naso-pharyngeal swabs, cross-contamination, and false negatives. The rate of qRT-PCR assay for naso- or oro-pharyngeal swabs positivity is $50-70 \%[22,23]$. Recent studies involving qRT-PCR assays with improved primer sets have much higher sensitivity and specificity. Chan et al. [24] developed and compared the performance of 3 novel RT-PCR assays targeting the RNA-dependent RNA polymerase $(\mathrm{RdRp}) /$ helicase (Hel), spike (S), and nucleocapsid (N) genes of SARS-CoV-2 with the RdRp-P2 assay, which is used in $>30$ European laboratories. The results showed that the COVID-19-RdRp/Hel assay had higher rates of positivity and did not cross-react with other human pathogenic coronaviruses and respiratory pathogens in cell culture and clinical specimens [24]. Yan et al. [25] developed a rapid, simple, specific, and sensitive reversetranscription loop-mediated isothermal amplification assay with primers specific for the replicase complex (orf1 $a b$ ) and Spike gene (S gene) of SARS-CoV-2 to detect viruses among clinical samples, which had $100 \%$ sensitivity and specificity with a mean $( \pm S D)$ time of $26.28 \pm 4.48$ min.

Antibody tests, such as the ELISA, colloidal gold immune chromatography, and chemiluminescence immune assay for immunoglobulin $\mathrm{M}(\mathrm{IgM})$ and immunoglobulin G (IgG) against SARS-CoV-2, improve the screening assay rates. Positive serum-specific IgM or IgG antibody titers in the recovery phase $\geq 4$ times higher than the acute phase can be used to diagnose patients with a negative qRT-PCR [14]. During follow-up monitoring, IgM and IgG are detectable 3-5 and 12 days after the symptom onset, respectively. Although false-positive and negative rates with antibody tests may be greater than the corresponding qRT-PCR rates [26]. Long et al. [27] report that within 19 days after symptom onset, $100 \%$ of patients tested positive for antiviral IgG. The detection efficiency by IgM ELISA is higher than qPCR 5.5 days after the symptom onset. The positive detection rate is significantly increased (98.6\%) when combining the IgM 
ELISA assay with PCR for each patient compared with a single qPCR test (51.9\%) [28]. Serologic testing may be helpful for the diagnosis of suspected patients with negative qRT-PCR results and the identification of asymptomatic infections; however, false-negative results with antibody tests in patients who are immunocompromised is concerning.

Transplant Candidates with Close Contact to Infected Persons or with a Positive qRT-PCT Test

The EBMT [17] and the American Society for Transplantation and Cellular Therapy (ASTCT) [29] recommend that transplant-related procedures be delayed 1421 days from the last contact and potential recipients be monitored for SARS-CoV-2 infection in patients if they had come in close contact to persons infected with SARS$\mathrm{CoV}-2$. In patients with advanced or high-risk disease and a positive qRT-PCR test, a transplant should be deferred until $\geq 2$ consecutive negative qRT-PCR tests $>1$ week apart. In patients with standard-risk disease, a 3 -month delay is recommended. These recommended intervals are not evidence based. Standard- or high-risk hematologic malignancies were classified on the basis of cytogenetic abnormalities, the white blood cell count at the time of diagnosis, response to induction chemotherapy, and relapse after the first complete remission.

\section{Transplant Recommendations}

\section{Allotransplant Recipients}

- Recipients should be managed in strict protective isolation. Visits from persons other than the designated caregiver should be discouraged.

- Healthcare providers with a temperature $>37.5^{\circ} \mathrm{C}$ or signs and symptoms consistent with SARS-CoV-2 infection should not come to work and should be screened by qRT-PCR.

- Recipients with a temperature $>37.5^{\circ} \mathrm{C}$ and cough or respiratory symptoms should undergo rapid testing for SARS-CoV-2 infection by qRT-PCR, serum antibody detection, and/or lung CT scan.

- Ideally, only one family member should care for and be in contact with the recipient. The designee should be tested for SARS-CoV-2 with qRT-PCR to detect asymptomatic infections, although there have been no published reported cases of transmission from caregivers.

- Educate recipients and family members on proper hand hygiene, mask wearing, and how to avoid potential contact risk behavior.

Management of Transplant during SARS-CoV-2
HLA-Matched Unrelated Donor Transplants

- If possible, stem cells from HLA-matched unrelated donors should be cryopreserved and shipped by local processing laboratories before starting pretransplant conditioning.

- Identify and evaluate an alternative HLA-haplotypematched related donor as a backup.

- Instruct donors of preventative measures.

\section{HLA-Identical or HLA Haplotype-Matched Related Transplants}

Identify and evaluate an alternative HLA haplotypematched related donor as a backup.

\section{Autotransplants}

After weighing the risks of delay and progression and considering alternative therapies, autotransplants should be deferred until after local resolution of the SARSCoV-2 pandemic if possible. If an autotransplant is urgent, we recommend testing the recipient for SARSCoV-2 infection as discussed above. For autologous stem cell mobilization and collection when COVID-19 is prevalent, a granulocyte colony-stimulating-factorbased regimen is preferred with minimal use of a chemotherapy regimen. Use of Mozobil ${ }^{\circledR}$ (plerixafor) depends on the pre-evaluation of hematopoietic function and the number of hematopoietic stem cells in the peripheral blood.

\section{Donor Recommendations}

Although data suggesting this practice are limited, when taking into account asymptomatic individuals infected with SARS-CoV-2, we advocate donor testing. Because the median incubation period of SARS-CoV-2 is 4 days (interquartile range, 2-7 days) [21], we recommend that donors be tested for SARS-CoV-2 infection using qRT-PCR, serum antibody detection, and lung CT scan 1 week before the proposed start of transplant conditioning. Donor access might be restricted by infection, logistics, and/or travel restrictions. Consequently, we advise the following:

- Minimize risk of infection by home isolation 14 days before the graft is obtained.

- If the donor has traveled to a SARS-CoV-2 endemic area or has been in close contact with persons travelling from such an area, the donor shall be excluded from donation for at least 14 days.

- If the donor has been in close contact with an individual diagnosed with SARS-CoV-2 infection, the donor

Acta Haematol 2021;144:500-506 
should be excluded from donation for at least 14 days and have a negative qRT-PCR test.

- Donors who are qRT-PCR-positive for SARS-CoV-2 should be excluded. Donors convalescing from infection with $\geq 2$ negative qRT-PCR tests may be taken into consideration for donation.

\section{Posttransplant Recommendations}

The interval immediately after discharge is the time of greatest risk for SARS-CoV-2 infection because of travel and exposure to infected individuals. We recommend self-isolation and minimal contact with family members. In the setting of a known high community prevalence of SARS-CoV-2 infections, nonessential critical clinic visits should be deferred or substituted with telemedicine consultations if possible. Reduce the use of public transport and find ways for blood tests to be done remotely, such as community hospitals, family health services, and commercial laboratories.

\section{Blood Donations}

Although SARS-CoV-2 RNA has been detected in blood samples from $1 \%$ of patients with COVID-19 [30], there are no reports of transmission by blood products. There are concerns that SARS-CoV-2 may be transmitted via blood products. We recommend that blood donors be tested for SARS-CoV-2 with qRT-PCR and serum antibody tests 1 week before donation.

\section{How to Treat HCT Recipients Infected with SARS-CoV-2}

Common symptoms of COVID-19, as reported by the Centers for Disease Control (CDC) and others, included fevers (44-98\%), dyspnea (19-64\%), cough (69-82\%), sputum (14-34\%), rhinorrhea (4-24\%), sore throat (5$14 \%)$, headaches (5-14\%), diarrhea (2-5\%), nausea/vomiting (1-10\%), and myalgias (11-15\%) [31]. Two transplant recipients with SARS-CoV-2 infections have been reported [11]. Transplant recipients are especially vulnerable to respiratory infections, and pathogen identification is always difficult, which complicates diagnosis and treatment. Transplant recipients with a temperature $>37.5^{\circ} \mathrm{C}$, cough, and other respiratory symptoms should be isolated and screened for respiratory pathogens based on po- tential risks. Transplant recipients might be at risk for major infections, including cytomegalovirus, EpsteinBarr virus, influenza virus $A$ and $B$, human herpes virus-6, herpes simplex virus, respiratory syncytial virus, and other pathogens. SARS-CoV-2 testing should be incorporated in the local workup.

Transplant recipients infected with SARS-CoV-2 should be placed in protective isolation. Recipients with COVID-19 should be treated according to local recommendations and with supportive care measures as needed, including mechanical ventilation and extracorporeal membrane oxygenation. There are no convincing data suggesting that chloroquine, hydroxychloroquine, azithromycin, intravenous immunoglobulin, antivirus drugs, or interleukin-6 antagonists are safe or effective. Corticosteroids are probably effective for patients with critically severe COVID-19; however, long-term use may be harmful [32].

Some data suggest that coagulation abnormalities occur in patients infected with SARS-CoV-2, especially those developing COVID-19, and include reactive thrombocytosis [21], disseminated intravascular coagulation [12, 33-36], and acroischemia [37]. Because allotransplant recipients are at risk for coagulation disorders, allotransplant recipients can develop complications, such as sinusoidal obstruction syndrome or thrombotic microangiopathy.

\section{Conclusions}

Social distancing, mask wearing, and education for patients and donors are essential preventative measures in these patients. We propose strategies to manage transplant-related activities during the SARS-CoV-2 pandemic (Fig. 1). Most of our recommendations are not evidence based, but are reasonable given current uncertainties and limitations in conducting controlled trials under the present circumstances. Our recommendations should be considered provisional and may require revision as more data become available.

\section{Acknowledgement}

R.P.G. acknowledges support from the National Institute of Health Research (NIHR) Biomedical Research Centre funding scheme.

\section{Conflict of Interest Statement}

The authors have no conflicts of interest to disclose. 


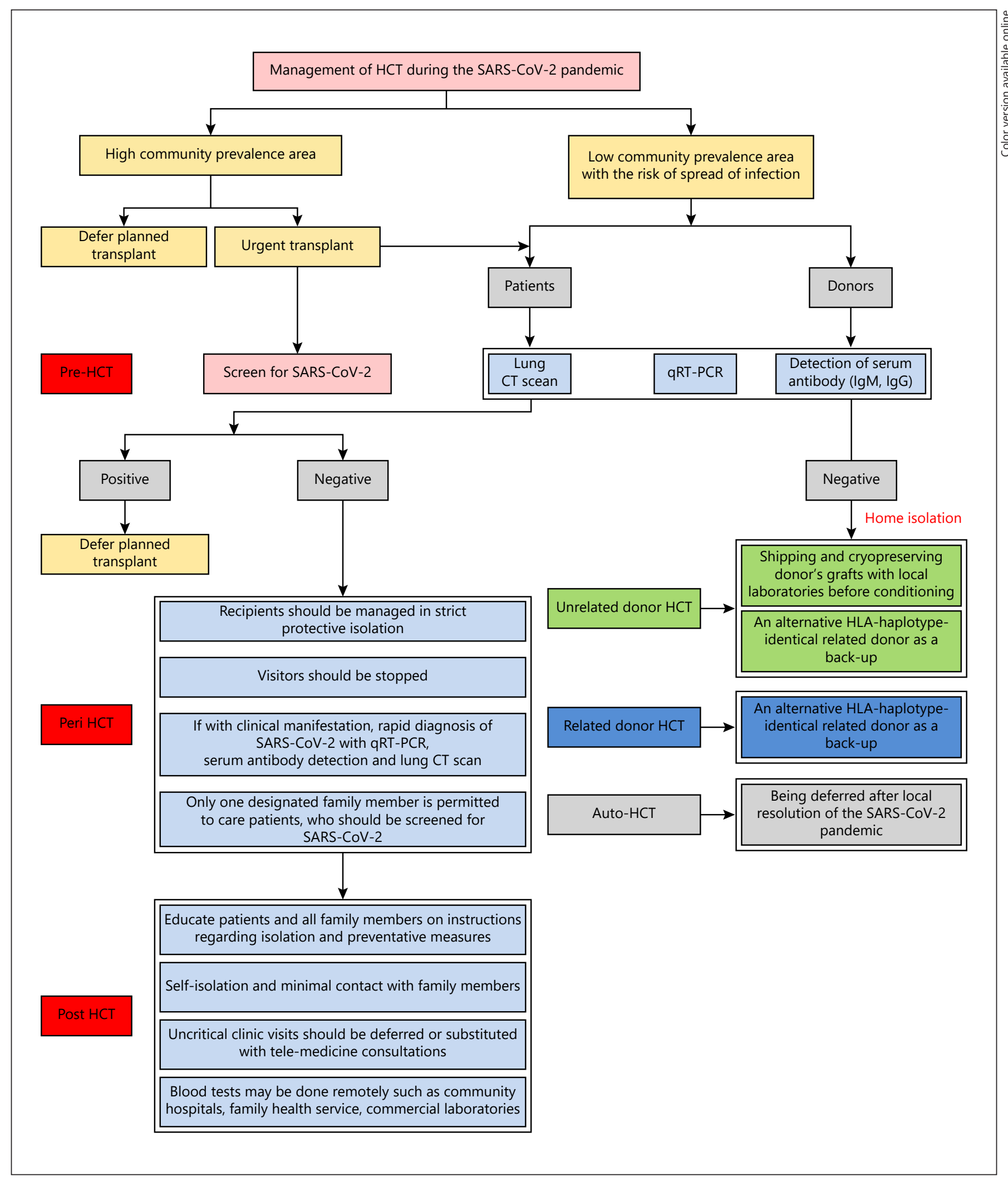

Fig. 1. Strategies to manage HCT during the SARS-CoV-2 pandemic. HCT, hematopoietic cell transplant; SARS$\mathrm{CoV}-2$, severe acute respiratory syndrome coronavirus-2. 


\section{Funding Sources}

Funded, in part, by the Key Project of the National Natural Science Foundation of China (81730008), the National Natural Science Foundation of China (81870136), and the Major Technology Program (Key Social Development) of the Science Technology Department of Zhejiang Province (2019C03016).

\section{Author Contributions}

Conception and design: L.Y., S.J., X.H., J.A., and H.H. Data collection, analysis, and interpretation: X.H. and H.H. Typescript preparation and revisions: X.H., M.M., R.P.G., A.N., and H.H.

\section{References}

1 Tomblyn M, Chiller T, Einsele H, Gress R, Sepkowitz K, Storek J, et al. Guidelines for preventing infectious complications among hematopoietic cell transplantation recipients: a global perspective. Biol Blood Marrow Transplant. 2009; 15(10):1143-238.

2 Liang W, Guan W, Chen R, Wang W, Li J, Xu K, et al. Cancer patients in SARS-CoV-2 infection: a nationwide analysis in China. Lancet Oncol. 2020;21(3):335-7.

$3 \mathrm{Yu}$ J, Ouyang W, Chua MLK, Xie C. SARSCoV-2 transmission in patients with cancer at a tertiary care hospital in Wuhan, China. JAMA Oncol. 2020;6:1108-10.

4 Kutikov A, Weinberg DS, Edelman MJ, Horwitz EM, Uzzo RG, Fisher RI. A war on two fronts: cancer care in the time of COVID-19. Ann Intern Med. 2020;172:756-8.

5 The Lancet Oncology. COVID-19: global consequences for oncology. Lancet Oncol. 2020;21: 467.

6 Xia Y, Jin R, Zhao J, Li W, Shen H. Risk of COVID-19 for patients with cancer. Lancet Oncol. 2020;21(4):e180.

7 He W, Chen L, Chen L, Yuan G, Fang Y, Chen $\mathrm{W}$, et al. COVID-19 in persons with haematological cancers. Leukemia. 2020;34(6):1637-45.

8 Li W, Wang D, Guo J, Yuan G, Yang Z, Gale $\mathrm{RP}$, et al. COVID-19 in persons with chronic myeloid leukaemia. Leukemia. 2020;34:17991804.

9 Robilotti EV, Babady NE, Mead PA, Rolling T, Perez-Johnston R, Bernardes M, et al. Determinants of severity in cancer patients with $\mathrm{CO}$ VID-19 illness. MedRxiv. Available from: https: //www.medrxiv.org/content/10.1101/2020.05.0 4.20086322v1.http: //dx.doi.org/10.1101/2020.0 5.04 .20086322

10 Chen Z, Xiong H, Li JX, Li H, Tao F, Yang YT, et al. [COVID-19 with post-chemotherapy agranulocytosis in childhood acute leukemia: a case report]. Zhonghua Xue Ye Xue Za Zhi. 2020;41: E004.

11 Wu T, Kang SC, Feng W, Fu H, Zhu XH, Wang $\mathrm{XJ}$, et al. [Biological characters analysis of $\mathrm{CO}$ VID-19 patient accompanied with aplastic anemia]. Zhonghua Xue Ye Xue Za Zhi. 2020;41: E003.

12 Mei H, Fang Y, Xia L. Recommendations for prophylaxis and prevention of COVID-19 in hematology department. Zhonghua Xue Ye Xue Za Zhi. 2020 Mar 11. Published online.

13 Jin XH, Zheng KI, Pan KH, Xie YP, Zheng MH. COVID-19 in a patient with chronic lympho- cytic leukaemia. Lancet Haematol. 2020;7(4): e351-2.

14 The seventh version of the clinical guidance for diagnosis and treatment for COVID-19 pneumonia issued by the National Health Commission of China. 2020 Mar 16.

15 Handbook of COVID-19 prevention and treatment. The first affiliated Hospital, Zhejiang University School of Medicine. Compiled According Clinical Experience. Available from: https:// covid-19.alibabacloud.com.

16 Novel Coronavirus Pneumonia Emergency Response Epidemiology. [The epidemiological characteristics of an outbreak of 2019 novel coronavirus diseases (COVID-19) in China]. Zhonghua Liu Xing Bing Xue Za Zhi. 2020;41: 145-51.

17 Available from: https://www.ebmt.org/ebmt/ news/coronavirus-disease-covid-19-ebmt-recommendations-update-march-16-2020.

18 Wang M, Wu Q, Xu W, Qiao B, Wang J, Zheng $\mathrm{H}$, et al. Clinical diagnosis of 8274 samples with 2019-novel coronavirus in Wuhan. medRxiv. 2020.

19 Bai Y, Yao L, Wei T, Tian F, Jin DY, Chen L, et al. Presumed asymptomatic carrier transmission of COVID-19. JAMA. 2020;323:1406-7.

20 D'Arienzo M, Coniglio A. Assessment of the SARS-CoV-2 basic reproduction number, R0, based on the early phase of COVID-19 outbreak in Italy. Biosaf Health. 2020 Jun;2:57-9.

21 Guan WJ, Ni ZY, Hu Y, Liang WH, Ou CQ, He JX, et al. Clinical characteristics of Coronavirus Disease 2019 in China. N Engl J Med. 2020;382: 1708-20.

22 Ai T, Yang Z, Hou H, Zhan C, Chen C, Lv W, et al. Correlation of chest CT and RT-PCR testing in Coronavirus Disease 2019 (COVID-19) in China: a report of 1014 cases. Radiology. 2020; 296:200642.

23 Yang Y, Yang M, Shen C, Wang F, Yuan J, Li J, et al. Evaluating the accuracy of different respiratory specimens in the laboratory diagnosis and monitoring the viral shedding of $2019-\mathrm{nCoV}$ infections. medRxiv. 2020.

24 Chan JF-W, Yip CC-Y, To KK-W, Tang TH-C, Wong SC-Y, Leung K-H, et al. Improved molecular diagnosis of COVID-19 by the novel, highly sensitive and specific COVID-19-RdRp/ Hel real-time reverse transcription-PCR assay validated in vitro and with clinical specimens. J Clin Microbiol. 2020;58(5):e00310.

25 Yan C, Cui J, Huang L, Du B, Chen L, Xue G, et al. Rapid and visual detection of 2019 novel coro- navirus (SARS-CoV-2) by a reverse transcription loop-mediated isothermal amplification assay. Clin Microbiol Infect. 2020;26:773-9.

26 Sun ZF, Meng XJ. Antigenic cross-reactivity between the nucleocapsid protein of severe acute respiratory syndrome (SARS) coronavirus and polyclonal antisera of antigenic group I animal coronaviruses: implication for SARS diagnosis. J Clin Microbiol. 2004;42(5):2351-2.

27 Long QX, Liu BZ, Deng HJ, Wu GC, Deng K, Chen YK, et al. Antibody responses to SARSCoV-2 in patients with COVID-19. Nat Med. 2020;26:845-8.

28 Guo L, Ren L, Yang S, Xiao M, Chang D, Yang $\mathrm{F}$, et al. Profiling early humoral response to diagnose Novel Coronavirus Disease (COVID-19). Clin Infect Dis. 2020;71:778-85.

29 Available from: https://www.astct.org.

30 Wang W, Xu Y, Gao R, Lu R, Han K, Wu G, et al. Detection of SARS-CoV-2 in different types of clinical specimens. JAMA. 2020;323:1843-4.

31 Pascarella G, Strumia A, Piliego C, Bruno F, Del Buono R, Costa F, et al. COVID-19 diagnosis and management: a comprehensive review. J Intern Med. 2020;288:192-206.

$32 \mathrm{Li} \mathrm{H}$, Chen C, Hu F, Wang J, Zhao Q, Gale RP, et al. Impact of corticosteroid therapy on outcomes of persons with SARS-CoV-2, SARS$\mathrm{CoV}$, or MERS-CoV infection: a systematic review and meta-analysis. Leukemia. 2020;34(6): 1503-11.

33 Tang N, Li D, Wang X, Sun Z. Abnormal coagulation parameters are associated with poor prognosis in patients with novel coronavirus pneumonia. J Thromb Haemost. 2020;18:844-847.

34 Chen N, Zhou M, Dong X, Qu J, Gong F, Han Y, et al. Epidemiological and clinical characteristics of 99 cases of 2019 novel coronavirus pneumonia in Wuhan, China: a descriptive study. Lancet. 2020;395(10223):507-13.

35 Huang C, Wang Y, Li X, Ren L, Zhao J, Hu Y, et al. Clinical features of patients infected with 2019 novel coronavirus in Wuhan, China. Lancet. 2020;395(10223):497-506.

36 Wang YD, Zhang SP, Wei QZ, Zhao MM, Mei $\mathrm{H}$, Zhang ZL, et al. [COVID-19 complicated with DIC: 2 cases report and literatures review]. Zhonghua Xue Ye Xue Za Zhi. 2020 Mar 14;41: 245-7.

37 Zhang Y, Cao W, Xiao M, Li YJ, Yang Y, Zhao J, et al. [Clinical and coagulation characteristics of 7 patients with critical COVID-2019 pneumonia and acro-ischemia]. Zhonghua Xue Ye Xue Za Zhi. 2020;41:E006. 